\title{
Redes neurales artificiales para estimar las pérdidas en la producción lechera
}

\author{
Borges, P.H.M. ${ }^{1} ;$ Mendoza, Z.M.S.H. ${ }^{2}$ y Morais, P.H.M. ${ }^{3}$
}

'Departamento de Suelos e Ingeniería Rural. Universidad Federal de Mato Grosso. Cuiabá. Brasil.

2Departamento de Ingeniería Forestal. Universidad Federal de Mato Grosso. Cuiabá. Brasil.

${ }^{3}$ Facultad de Agronomia y Zootecnia. Universidad Federal de Mato Grosso. Cuiabá. Brasil.

PalABRAS CLAVE ADICIONALES

Desempeño animal.

Inteligencia artificial.

Conforto ambiental.

\begin{abstract}
RESUMEN
Este estudio tuvo como objetivo desarrollar redes neurales artificiales para estimar las pérdidas en la producción lechera, en función del índice de temperatura y humedad (ITU), considerándose el día del año. Accediendo al sitio del Instituto Nacional de Meteorología, se obtuvieron los valores de temperatura y humedad para una serie histórica de 27 años en el municipio de Nova Xavantina-MT. Así, se determinó el índice de temperatura y humedad máximo en cada día del año durante el referido período. Se optó por las redes de múltiples camadas del tipo "perceptron". Se varió el número de camadas y neuronas para formar diferentes arquitecturas. La proximidad entre los valores reales y los estimados se evaluó por medio de diversos criterios estadísticos y por los coeficientes ofrecidos por el propio programa computacional utilizado. De esta forma se seleccionó la rede con mejor desempeño. Luego se concluyó que las redes presentaron adecuada confiablidad y precisión para predecir el ITU, de acuerdo con el día del año y que ellas pueden ser una herramienta útil en el manejo de vacas lecheras y en el planeamiento de medidas para amenizar el ambiente térmico.
\end{abstract}

\section{Artificial neural networks to estimate the losses in dairy production}

\section{SUMMARY}

This study aimed to develop artificial neural networks to estimate losses in milk production, as function of the temperature and humidity index (ITU) by considering of the year day. Accessing the site of the National Institute of Meteorology, temperature and humidity values obtained for a historical series of 27 years in the township of Nova Xavantina-MT. Thus, the maximum temperature and humidity index was determined on each year day during the said period. We chose multi-layer networks of the "perceptron" type. The number of layers and neurons varied to form different architectures. The proximity between the real and the estimated values evaluated by means of various statistical criteria and by the coefficients offered by the used computational program. In this way, selected the network with the best performing. It was then concluded that the networks presented adequate reliability and precision to predict ITU, according to the year day and that they can be a useful tool for the management of dairy cows and for the planning of providences to soften the thermal environment.

\section{INTRODUCCIÓN}

En las últimas décadas, el control del ambiente de instalaciones zootécnicas en términos de bien estar animal ha sido una constante preocupación, pues alteraciones fisiológicas en respuesta al excesivo calor pueden causar grandes perjuicios a los productores. Estudios han revelado la sensibilidad de los animales al estrés térmico y la considerable disminución del desempeño productivo y reproductivo, siendo más acentuado durante el verano, cuando la temperatura y la humedad del aire alcanzan los valores máximos (Perissinotto et al. 2007; Souza et al. 2010). De acuerdo con esos autores, las vacas lactantes reducen la ingestión de materia seca para amenizar el incremento calórico, lo que trae como consecuencia reducción en la producción de leche.

El impacto de factores ambientales en la producción animal ha sido evaluado por medio de diversos índices de conforto térmico y son estimados, en función de la 
temperatura, la humedad relativa del aire, la velocidad del viento y la radiación solar. El índice de temperatura y humedad (ITU) es el más utilizado para expresar el conforto animal en relación al ambiente. De esta forma, a partir de mediciones de las variables climatológicas temperatura y humedad relativa del aire realizadas en las instalaciones zootécnicas o en sus proximidades, se puede determinar el referido índice. Los datos pueden servir como apoyo a la toma de decisión, en un intento de aliviar el estrés por calor en los animales. También, el estudio de ese índice puede contribuir en la definición de padrones de condiciones ambientales y en la elaboración de un modelo empírico de previsión.

Los modelos empíricos para previsión de variables meteorológicas pueden ser ajustados a los dados por medio de distribuciones probabilísticas. Sin embargo, uno de los principales inconvenientes en los modelos de simulación que considera ese tipo de variable se encuentra en la incapacidad de reconocer su forma real, es decir, ignorar o no distinguir la inestabilidad del tiempo de duración de una determinada condición. Para superar esas deficiencias en los modelos matemáticos y estadísticos tradicionales, Bilgili \& Sahin (2010) y Wu et al. (2010), utilizaron redes neurales artificiales. Esos autores destacan, que los modelos de simulación con variables climáticas deben poseer una gran cantidad de datos observados para ser confiables.

La aplicación de redes neurales artificiales (RNAs) ha sido propuesta por varios autores para modelar la precipitación, la evapotranspiración y la humedad (Alves Sobrinho et al. 2011; Carvalho et al. 2012; Yasar et al. 2012). También, Santos et al. (2016) utilizaron redes neurales artificiales del tipo perceptron con múltiples camadas para monitorear cerdos y obtuvieron resultados precisos y con adecuada exactitud. Esos estudios muestran la viabilidad del uso de los referidos modelos y su habilidad para la previsión de dados con base en informaciones anteriores. Las redes artificiales son modelos semejantes a las estructuras neurales bilógicas y tienen capacidad computacional adquirida por medio de aprendizaje y generalización.

Con base en los estudios consultados se infiere que las referidas redes constituyen una poderosa y viable herramienta computacional para el procesamiento de datos y que pueden ser muy útiles para estimar índices de conforto térmico a partir de valores de variables climáticas registrados en las proximidades de las instalaciones zootécnicas. Así, esos modelos deben proporcionar los fundamentos necesarios para caracterizar el ambiente térmico y de esa forma auxiliar en el manejo de los animales. Esta problemática motivo la elaboración del presente trabajo, que tiene por objetivo estimar las pérdidas en la producción lechera, en función del índice de temperatura y humedad (ITU), aplicándose redes neurales artificiales.

\section{MATERIAL Y MÉTODOS}

En este estudio se utilizaron informaciones meteorológicas provenientes del BDMEP (Banco de Datos Meteorológicos para Enseñanza e Investigación), disponibles por el INMET (Instituto Nacional de Meteorología). Se optó por la Estación Climatológica Con- vencional del municipio Nova Xavantina-MT, código OMN 83319, coordenadas geográficas 140 42' 00" de Latitud Sur y $52021^{\prime} 00^{\prime \prime}$ de Longitud Oeste, altitud de 316 m y operante desde 05/08/1987. Los datos se refieren al período entre 01/01/1988 y 31/12/2015, totalizando 27 años. La región presenta un clima tropical, según Peel et al. (2007) clasificado como Aw, con temperatura media anual de 25,1 0C y pluviosidad media anual de 1498 mm (INMET 2017).

Accediendo a la base de datos BDMEP se colectaron los valores de temperatura de bulbo seco y humedad relativa para las series históricas de datos horarios correspondientes a 00:00; 12:00 y 18:00 horas. Los datos se almacenaron debidamente en hojas de cálculo con auxilio de la planilla electrónica Microsoft EXCEL. Después, esos valores se tabularon y organizaron, de acuerdo con el día y horario, totalizando cerca de 1095 lecturas por año. Para el tratamiento y procesamiento de los datos, así como para definir la arquitectura de las redes neuronales artificiales se utilizó el programa estadístico STATISTICA 8.0.

En el análisis del índice de temperatura y humedad (ITU), se utilizó la clasificación propuesta por Rosenberg et al. (1983), que considera conforto normal para ITU inferior a 74, ITU entre 74 y 78 alerta a los productores, es decir, deben ser tomadas algunas medidas para evitar pérdidas, ITU en el intervalo de 78 a 84 indica peligro, o sea que, las pérdidas son inevitables y cuando el ITU sea mayor que 84 es señal de emergencia, comprometiéndose la salud del animal. Así, el conforto ambiental se evaluó por el índice de temperatura y humedad (ITU), sugerido por Buffington et al. (1981), dado por:

$\mathrm{ITU}=\mathrm{Tbs}+0,36 \cdot \mathrm{Tpo}+41,2$

En que,

ITU: Índice de temperatura y humedad (adimensional);

Tbs: Temperatura de bulbo seco o ambiente ( $\left.{ }^{\circ} \mathrm{C}\right)$;

Tpo: Temperatura del punto de condensación $\left({ }^{\circ} \mathrm{C}\right)$.

Los valores del índice de temperatura y humedad estimados se analizaron inicialmente por estadísticas descriptivas con el objetivo de caracterizar la distribución de los datos. Luego, se determinó la media, valores mínimos y máximos, desvío padrón, coeficiente de variación e intervalo de confianza para 95\% de probabilidad. La declinación en la producción lechera se estimó, en función del nivel de producción normal (PN). Esa variable es una referencia de forma genérica, considerándose una situación en que el animal no esté sometido al estrés térmico, es decir, la productividad que tendría para una condición de termoneutralidad. En este trabajo se adoptaron los niveles normales de producción 10, 15, 20, 25, 30 y $35 \mathrm{~kg} \mathrm{vaca}{ }^{-1}$ día $^{-1}$, así como la expresión adaptada por Hahn (1993):

$\mathrm{DPL}=-1,075-1,736 \cdot \mathrm{PN}+0,02474 \cdot \mathrm{PN} \cdot \mathrm{ITU}$

En que,

DPL: Declinación en la producción lechera $\left(\mathrm{kg} \mathrm{dia}^{-1}\right)$;

PN: Nivel normal de producción $\left(\mathrm{kg} \mathrm{dia}^{-1}\right)$; 
ITU: Índice de temperatura y humedad (adimensional).

Basado en los trabajos de Terra \& Passador (2012), Ventura et al. (2012), Georgens et al. (2014) y Valente et al. (2014), se optó por las redes neurales artificiales con la estructura "Perceptrons" de múltiples camadas (MPL). Para definir la arquitectura se varió el número de camadas intermediarias y el número de neuronas en las camadas, conforme los criterios de Terra \& Passador (2012) y Ventura et al. (2012). Como variable de entrada se introdujo el día, siendo la variable de salida el índice de temperatura y humedad (ITU). En todas las arquitecturas se utilizó la función logística para activar las redes. Las camadas se conectaron por sinapsis completas, utilizándose la retro propagación del erro como algoritmo de aprendizaje.

Para reducir el número de redes obtenidas y seleccionar las más adecuadas, se utilizaron los propios coeficientes e índices del programa correspondientes al entrenamiento, selección y validación, así como se determinó el error medio y la raíz del error cuadrático medio (Georgens et al. 2014). Las redes neuronales clasificadas como satisfactorias se analizaron de forma más rigurosa. Ese procedimiento contó con la elaboración del gráfico de dispersión e histograma de residuos y con la aplicación de las pruebas Kolmogorov-Smirnov, Lilliefors, Shapiro-Wilk, Chi Cuadrado y t-Student entre los valores observados y los estimados. Después, se calcularon los coeficientes de correlación y concordancia de Willmott para evaluar la precisión y la exactitud de las redes. Luego, se multiplicaron esos dos coeficientes e se obtuvo el índice de desempeño para evaluar finalmente la eficiencia, pues este último mide la proximidad entre los valores estimados por las redes y los observados.

\section{RESULTADOS Y DISCUSIÓN}

La Tabla I contiene las principales estadísticas descriptivas del índice de temperatura y humedad (ITU) para cada mes, obtenidas de las 18436 observaciones provenientes da serie histórica de 30 años. En esa Tabla se puede constatar que los valores medios del referido índice se mantuvieron en el intervalo de alerta establecido por Rosenberg et al. (1983), independiente del mes. En la propia Tabla se observa que los mínimos valores siempre fueron inferior a 72 , sin embargo, los máximos indicaron estado de emergencia en por lo menos un día de cada mes. El desvío padrón superior a 3,5 unidades nos muestra elevada dispersión en relación a la media, llevando en consideración que las fajas de alerta y peligro tienen una amplitud de 4 unidades. Esos valores concuerdan con lo esperado y confirman la alta variabilidad de los factores climáticos durante todo el año, principalmente la temperatura y la humedad relativa.

La precisión de las observaciones, con base en el coeficiente de variación, se consideró media. Sin embargo, los cortos intervalos de confianza obtenidos, cuya mayor amplitud fue de 0,6 durante los meses de julio y agosto indican adecuada precisión en la determinación del índice de temperatura y humedad (ITU) para el nivel de $95 \%$ de probabilidad (Tabla I). El referido intervalo depende del número de observaciones, el cual fue superior a 1500 en todos los meses, eso representa aproximadamente 50 lecturas para cada día. Esos resultados permiten afirmar con suficiente confiabilidad que el índice de temperatura y humedad se mantuvo clasificado como alerta durante la mayor parte del año, es decir, solo los meses de junio, julio y agosto ofrecieron condiciones de conforto térmico (Tabla I).

Se debe destacar que los coeficientes de variación mensuales obtenidos en este trabajo fueron superiores al valor de 3,64\% determinado por Souza et al. (2016) para el índice de temperatura y humedad en seis microrregiones del estado de Paraíba. Estas discrepancias tienen como causa principal el universo de datos utilizados en cada investigación. En este estudio se analizó el índice de temperatura y humedad diario correspondiente a tres horarios para una serie histórica

Tabla I. Estadísticas descriptivas del índice de temperatura y humedad para cada mes (Descriptive statistics of the temperature and humidity index for each month).

\begin{tabular}{lcccccc}
\hline \multirow{2}{*}{ Meses } & \multicolumn{7}{c}{ Estadística descriptiva } \\
\cline { 2 - 7 } & Media & Mínimo & Máximo & Desvío Padrón & Coeficiente de variación (\%) & Intervalo de confianza (95\%) \\
\hline Enero & 75,87 & 68,83 & 88,23 & 3,54 & 4,66 & $\pm 0,18$ \\
Febrero & 76,09 & 68,86 & 86,63 & 3,62 & 4,76 & $\pm 0,18$ \\
Marzo & 76,29 & 68,01 & 86,46 & 3,76 & 4,93 & $\pm 0,19$ \\
Abril & 76,37 & 62,39 & 86,42 & 4,14 & 5,42 & $\pm 0,21$ \\
Mayo & 74,21 & 59,61 & 85,79 & 5,09 & 6,86 & $\pm 0,25$ \\
Junio & 72,49 & 61,89 & 85,58 & 5,78 & 7,98 & $\pm 0,29$ \\
Julio & 72,01 & 59,62 & 85,40 & 6,07 & 8,43 & $\pm 0,30$ \\
Agosto & 73,56 & 58,13 & 88,40 & 5,99 & 8,15 & $\pm 0,30$ \\
Septiembre & 76,70 & 63,58 & 90,18 & 5,27 & 6,87 & $\pm 0,27$ \\
Octubre & 77,62 & 68,17 & 90,44 & 4,45 & 5,74 & $\pm 0,22$ \\
Noviembre & 76,63 & 68,76 & 88,08 & 3,74 & 4,88 & $\pm 0,19$ \\
Diciembre & 76,02 & 68,50 & 87,98 & 3,52 & 4,51 & $\pm 0,17$ \\
Anual & 75,62 & 58,13 & 90,44 & 4,51 & 5,97 & $\pm 0,26$ \\
\hline
\end{tabular}


de 30 años, mientras en el citado anteriormente, ese coeficiente correspondió a los valores medios diarios de cuatro años, lo que pudo reducir la variabilidad de los datos.

El valor medio anual del índice de temperatura y humedad igual a 75,62 estimado en este trabajo, muestra situación de alerta en relación al conforto térmico para vacas lecheras en el municipio de Nova Xavantina-MT. Estos resultados son semejantes a los obtenidos por Souza et al. (2016) en tres microrregiones del estado de Paraíba: Alto Sertão, Sertão y Litoral, siendo los valores del referido índice 75,61; 76,41 y 76,62, respectivamente. También, Lima et al. (2006) determinaron un valor medio anual de ITU igual a 74,6 en 2002 para la región lechera del Valle de Paraíba-SP, caracterizando una situación sin estrés térmico, pero de alerta. Turco et al. (2006) relataron estado de alerta en la mayor parte del Estado de Bahía durante los meses más calientes del año con valores medios de ITU entre 73 y 77. Con base en esos trabajos se puede afirmar que el ITU debe ser monitoreado diariamente, pues las variables temperatura y humedad presentan elevada amplitud durante períodos largos (meses y año), siendo los dados medios insuficientes para garantizar el conforto ambiental adecuado de las vacas lecheras.

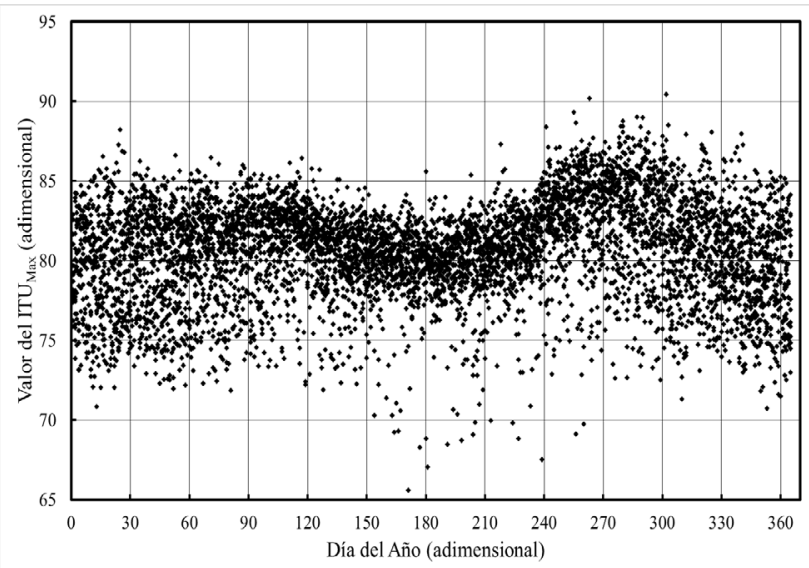

Figura 1. Diagrama de dispersión con los valores del índice de temperatura y humedad máximo, en función del día del año para el municipio de Nova XavantinaMT (Dispersion diagram with values of maximum temperature and humidity index, as a function of the day of the year for the municipality of Nova Xavantina-MT).
Con el objetivo de caracterizar la distribución del índice de temperatura y humedad para el municipio de Nova Xavantina-MT, en el presente estudio se optó por representar el máximo valor del referido atributo, en función del día del año (Figura 1). En esa Figura se verifica que, en muchos días, principalmente, durante los meses de septiembre y octubre el ITU alcanzó la categoría de emergencia, es decir fue mayor que 84, pudiendo llevar a pérdidas considerables en la producción lechera e inclusive perjudicar la salud de los animales, de acuerdo con relatos de Rodrigues et al. (2010), Almeida et al. (2013), Ricci et al. (2013) y Souza \& Batista (2012). También, en la misma Figura se puede constatar que la grande mayoría de valores de ITU se concentró en la faja de peligro durante todo el año, es decir, entre 78 y 84 . Estos resultados demuestran que la previsión diaria del ITU puede ser de gran valor para planear e definir acciones de manejo del rebaño con el propósito de amenizar el ambiente térmico.

De acuerdo con los criterios de la bibliografía consultada, se definieron y probaron diferentes arquitecturas de redes conteniendo hasta dos camadas intermediarias u ocultas y cada una teniendo como máximo ocho neuronas, siendo evaluadas un total de 26 combinaciones. Sin embargo, solo seis presentaron índices satisfactorios, dados por la pequeña diferencia entre los parámetros obtenidos por medio del programa, es decir los índices de entrenamiento, selección y prueba (Tabla II). En esa Tabla se observa que los referidos índices están muy próximos, lo que muestra un eficiente desempeño de las redes neurales artificiales, así como una adecuada proporción en el número de elementos que componen los conjuntos de dados para las etapas de entrenamiento, selección y prueba.

También, en la Tabla II se puede confirmar que los menores valores para el error medio y la raíz del error cuadrático medio correspondieron a la primera rede neural artificial, la cual está compuesta por una neurona de entrada e otra de salida, así como una camada oculta con cuatro neuronas. Por otro lado, esa arquitectura presentó el mayor coeficiente de determinación $\left(R^{2}\right)$, siendo inclusive superior a 0,81 , valor mínimo recomendado por Georgens et al. (2014) para indicar una fuerte relación entre los valores observados y los estimados. Con base en la Tabla II se deduce que el aumento del número de camadas ocultas y de neuronas no interfirió en la precisión, pues los mejores índices se obtuvieron con la rede 1 que posee una camada oculta.

Tabla II. Valores de los índices evaluados para cada arquitectura de rede neuronal artificial (Values of the evaluated indexes for each artificial neural network architecture).

\begin{tabular}{|c|c|c|c|c|c|c|c|}
\hline \multirow{2}{*}{ RNA } & \multirow{2}{*}{ Arquitectura } & \multicolumn{3}{|c|}{ Parámetros del programa } & \multirow{2}{*}{ Error medio } & \multirow{2}{*}{$\mathrm{R}^{2}$} & \multirow{2}{*}{ RMSE } \\
\hline & & Entrenamiento & Selección & Prueba & & & \\
\hline 1 & MLP 1:1-4-1:1 & 1,0021 & 0,9983 & 0,9976 & 0,0417 & 0,8359 & 4,6378 \\
\hline 2 & MLP 1:1-7-1:1 & 0,9218 & 0,9520 & 0,9508 & $-0,0449$ & 0,6251 & 5,1763 \\
\hline 3 & MLP 1:1-2-1-1:1 & 0,9269 & 0,9600 & 0,9475 & $-0,0554$ & 0,6038 & 5,2961 \\
\hline 4 & MLP 1:1-2-6-1:1 & 0,9363 & 0,9232 & 0,9586 & 0,0563 & 0,5712 & 5,3742 \\
\hline 5 & MLP 1:1-6-4-1:1 & 0,9759 & 0,9968 & 1,0326 & 0,0642 & 0,5461 & 5,5746 \\
\hline 6 & MLP 1:1-8-1-1:1 & 0,8397 & 0,9347 & 0,9343 & $-0,0678$ & 0,5034 & 6,7314 \\
\hline
\end{tabular}




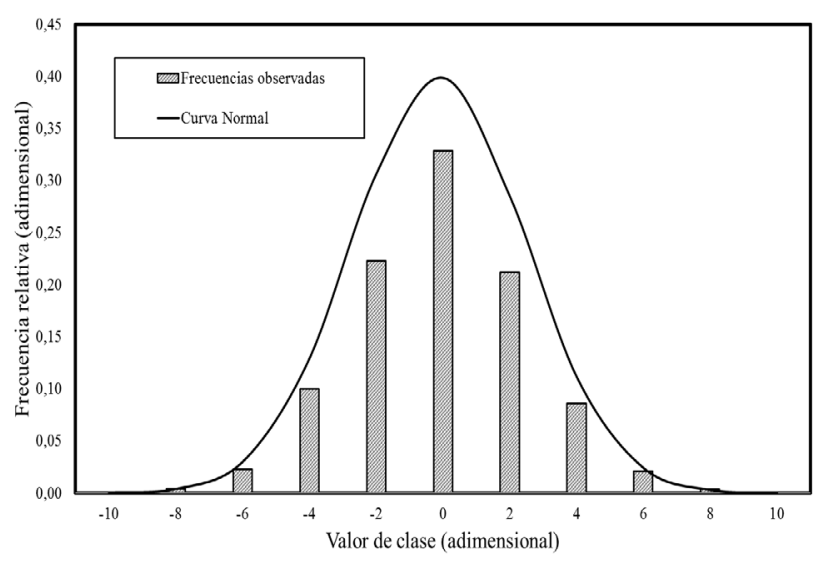

Figura 2. Histograma de frecuencia relativa de los residuos y curva normal para la rede neural artificial 1 (Histogram of relative frequency of residues and normal curve for the artificial neural network 1).

Estos resultados no concuerdan con las sugestiones de Terra \& Passador (2012), respecto al número de neuronas en las camadas, pues ninguno de los criterios propuestos se adecuó a los dados del presente estudio. No obstante, se confirmaron las recomendaciones de Georgens et al. (2014). Esos autores afirman que no existe un criterio preciso para definir el número de neuronas en las camadas, siendo necesario la prueba y verificación de diversas redes por medio de tentativas. Considerándose que la rede neuronal artificial 1 proporcionó los indicadores más apropiados, solamente esa arquitectura fue objeto del análisis complementar gráfico y analítico para verificar su eficiencia y confiabilidad. Así, inicialmente se elaboraron los histogramas de frecuencia y el diagrama de los residuos como función del índice de temperatura y humedad estimado, ilustrados en las Figuras 2 y 3 , respectivamente.

Con base en la Figura 2 se deduce que las diferencias entre el índice de temperatura y humedad medido

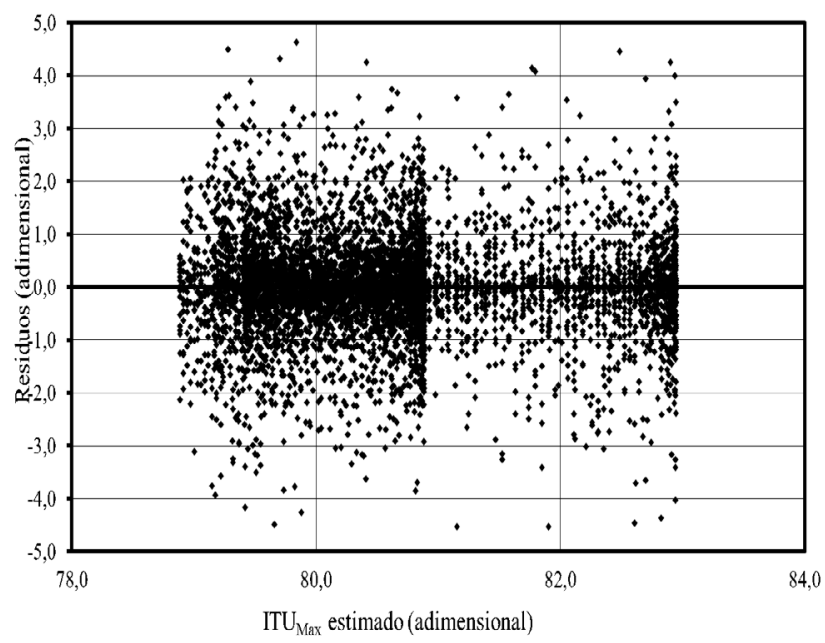

Figura 3. Valores de los residuos, en función del índice de temperatura y humedad máximo estimado para la rede neural artificial 1 (Values of the residues, as a function of the maximum temperature and humidity index estimated for the artificial neural network 1). y el estimado por las redes neuronales se distribuyeron con adecuada simetría y próximos de la curva normal, es decir, la mayoría de los residuos de pequeña magnitud se concentraron en el centro, alrededor de cero y los restantes con valores más elevados en los extremos. Esa característica del histograma de frecuencia en relación a la normal constituye una premisa fundamental para definir criterios sobre la eficiencia en la predicción de las redes. También, de acuerdo con la Figura 3 se infiere que no hay relación entre los residuos y el día del año que fue la variable independiente considerada en la rede elaborada, pues los puntos se situaron aleatoriamente a lo largo del eje horizontal y no existen evidencias de algún padrón de dependencia funcional.

Aunque la distribución de los residuos presentó características de normalidad y no se evidenció padrón de dependencia funcional en los residuos, el análisis gráfico se considera un procedimiento subjetivo. Luego, se verificó analíticamente la normalidad, conforme las pruebas de Kolmogorov-Smirnov, Lilliefors, Shapiro-Wilk y de adherencia Chi Cuadrado. Además, se aplicó la prueba de t-Student para constatar que no hubo diferencias entre los valores medidos y los estimados, bien como se comprobó la eficiencia de las redes con base en el índice de desempeño. Los resultados de esas pruebas y criterios estadísticos constan en la Tabla III. De acuerdo con la Tabla III, los residuos oriundos de la rede neuronal artificial 1 (MLP 1:14-1:1) evidenciaron normalidad. Esa característica le confiere mayor confiabilidad a la rede, tomando como referencia los estudios de Torman et al. (2012) y Lopes et al. (2013).

También, la prueba t-Student indicó que no existen diferencias entre los máximos valores del índice de temperatura y humedad medidos y los estimados por la rede (Tabla III). En esa Tabla se puede constatar que el coeficiente de correlación y el de concordancia de Willmott superaron el valor mínimo 0,7, sugerido por Terra \& Passador (2012) y Georgens et al. (2014). Según los criterios de Paiva \& Souza (2016), la precisión y la exactitud de la rede neural se clasificó, respectivamente, como muy buena y excelente, siendo considerada muy buena la calidad del ajuste (Tabla III). Así, se confirma la adecuada confiabilidad de la rede desarrollada, lo que justifica su aplicación para fines de planeamiento y manejo del rebaño lechero. En la Figura 4 se representa la declinación de la producción lechera estimada por la rede neural, en función del día del año para cada nivel estudiado. Se puede constatar que las pérdidas son mayores a medida que aumenta el nivel de producción, acentuándose durante los meses de septiembre y octubre (Figura 4). Esa tendencia concuerda con los resultados obtenidos por Lima et al. (2006), Turco et al. (2006) y Souza et al. (2016). Considerándose que la rede permite estimar el índice de temperatura y humedad para cualquier día del año, la misma puede auxiliar en el planeamiento de medidas simples para amenizar el ambiente térmico de las vacas lecheras. Como alternativas prácticas y de bajo costo, Rodrigues et al. (2010), Bertoncelli et al. (2013) y Cerutti et al. (2013) relatan que el sombreado natural o artificial y la aspersión de agua en las instalaciones de ordeña contribuyen significativamente para mejorar los resul- 
Tabla III. Resultados de las pruebas y criterios estadísticos aplicados a la rede neural artificial 1 (Results of the tests and statistical criteria applied to the artificial neural network 1).

\begin{tabular}{lccc}
\hline Prueba o criterio & Tipo & Estadística & Resultado \\
\hline Kolmogorov Smirnov & Normalidad & $0,0981(p>0,2)$ & no significativo \\
Lilliefors & Normalidad & $\mathrm{P}>0,2$ & no significativo \\
Shapiro Wilk & Normalidad & $0,9584(\mathrm{p}=0,0583)$ & no significativo \\
Chi Cuadrado & Adherencia & $17,05(\mathrm{p}=0,0732)$ & no significativo \\
Student $(\mathrm{t})$ & Hipótesis & $1,42(\mathrm{p}=0,0781)$ & no significativo \\
Coeficiente de correlación & Precisión de la RNA & 0,9142 & Muy buena \\
Coeficiente de Willmott & Exactitud de la RNA & 0,9435 & Excelente \\
Índice de desempeño & Calidad del ajuste & 0,8625 & Muy buena \\
\hline
\end{tabular}

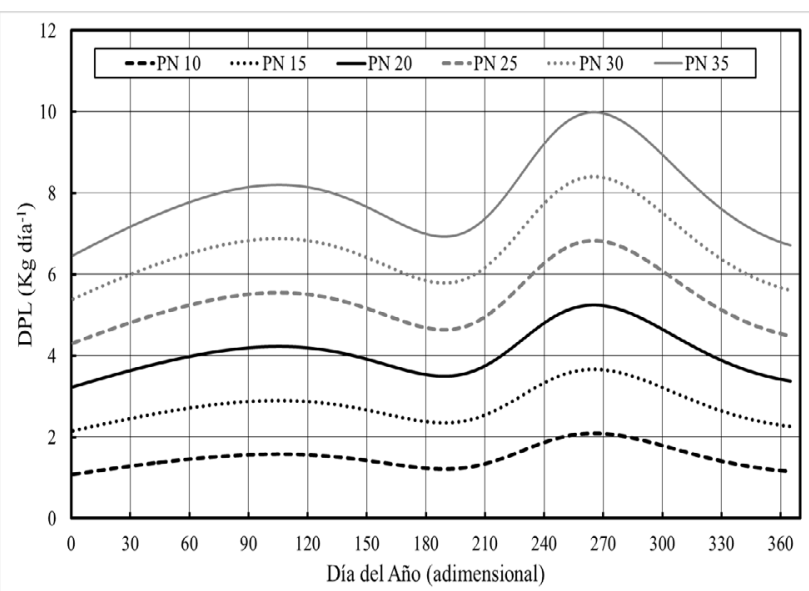

Figura 4. Declinación de la producción lechera estimada por la rede, en función del día del año (Declining of milk production estimated by the network, depending on the day of the year).

tados productivos. Conforme Nara et al. (2016), el lucro da la propiedad puede aumentar 1,15 reales por cada litro de leche, evidenciando la importancia de elevar la producción.

\section{CONCLUSIÓN}

Las redes neuronales artificiales de múltiples camadas del tipo perceptrón estimaron satisfactoriamente el índice de temperatura y humedad. También, fue posible definir y verificar una arquitectura apropiada para determinar el índice de temperatura y humedad, en función del día del año. Las redes desarrolladas presentaron adecuada confiabilidad y precisión en las predicciones, lo que justifica su aplicación. Las redes neuronales artificiales obtenidas pueden ser una herramienta útil en el manejo de vacas lecheras y en el planeamiento de medidas para amenizar el ambiente térmico de las salas de ordeña.

\section{AGRADECIMIENTOS}

Los autores agradecen al Instituto Nacional de Meteorología (INMET) por permitir utilizar el banco de datos para enseñanza e investigación de las estaciones meteorológicas en el Estado de Mato Grosso.

\section{BIBLIOGRAFÍA}

Almeida, GLP, Pandorfi, H, Barbosa, SBP, Pereira, DF, Guselini, C \& Almeida, GAP 2013, 'Comportamento, produção e qualidade do leite de vacas Holandês-Gir com climatização no curral', Revista Brasileira de Engenharia Agrícola e Ambiental, vol. 17, num. 8, p. 892-899.

Alves Sobrinho, T, Rodrigues, DBB, Oliveira, PTS, Rebucci, LCS \& Pertussatti, CA 2011, 'Estimativa da evapotranspiração de referência através de redes neurais artificiais', Revista Brasileira de Meteorologia, vol. 26, num.2, p. 197-203.

Bertoncelli, P, Martin, TN, Ziech, MF, Paris, W \& Cella, PS 2013, 'Conforto térmico alterando a produção leiteira', Enciclopédia Biosfera, vol. 9, num. 17, p. 762-777.

Bilgili, M \& Sahin, B 2010, 'Comparative analysis of regression and artificial neural network models for wind speed prediction', Meteorology and Atmospheric Physics, vol. 109, num. 1, p. 61-72.

Buffington, DE, Collier, R \& Canton, GH 1981, 'Black globe-humiddity inde (BGHI) as confort equation for dairy cows', Transactions of the $A S A E$, vol. 24, num. 3, p. $711-714$.

Carvalho, DF, Khoury Júnior, JK, Varella, CAA, Giori, JZ \& Machado, RL 2012, 'Rainfall erosivity for the state of Rio de Janeiro estimated by artificial neural network', Revista Engenharia Agrícola, vol. 32, num. 1, p. 197-207.

Cerutti, WG, Bermudes, RF, Viegas, J \& Martins, CMMR 2013, 'Respostas fisiológicas e produtivas de vacas holandesas em lactação submetidas ou não a sombreamento e aspersão na pré-ordenha', Revista Brasileira de Saúde e Produção Animal, vol. 14, num. 3, p. $406-412$.

Georgens, EB, Leite, HG, Gleriani, JM, Soares, CPB \& Ceolin, A 2014 , 'Influência da arquitetura na estimativa de volume de árvores individuais por meio de redes neurais artificiais', Revista Árvore, vol. 38 , num. 2 , p. 289-295.

Hahn, G 1993,'Bioclimatologia e instalações zootécnicas: aspectos teóricos e aplicados'. FUNEP, 28p.

Instituto Nacional de Meteorologia (INMET). Banco de dados meteorológicos para ensino e pesquisa.

Disponível em: <http://www.inmet.gov.br/portal/index.php?r=bdmep/bdmep>. Acesso em: 20 janeiro 2017.

Lima, KAO, Moura, DJ, Nääs, IA, Perissinoto, M \& Souza, SRL 2006, 'Avaliação bioclimatológica da bacia leiteira do vale do Paraíba-SP utilizando o ITU', Veterinária Notícias, vol. 12, num. 2, p. 31.

Lopes, MM, Castelo Branco, VTF \& Soares, JB 2013, 'Utilização dos testes estatísticos de Kolmogorov-Smirnov e Shapiro-Wilk para verificação da normalidade para materiais de pavimentação', Revista Transportes, vol. 21, num. 1, p. 59-66.

Nara, EOB, Guerra, T, Xavier, MB, Kipper, LM, Benitez, LB \& Moraes, J 2016, 'Analysis of milk production at the Taquari Valley, Rio Grande do Sul, Brazil', Archivos de Zootecnia, vol. 65, num. 252 , p. 555-562. 
Paiva, CM \& Souza, ASP 2016, 'Avaliação de métodos de estimativa da evapotranspiração de referência para fins de manejo da irrigação', Anuário do Instituto de Geociências - UFRJ. vol. 39, num. 1, p. 42-51.

Peel, MC, Finlayson, BL \& Mcmahon, TA 2007, 'Updated world of the Köppen-Geiger climate classification', Hydrology and Earth System Sciences, vol. 11, p. 1633-1644.

Perissinotto, M, Cruz, VF, Pereira, A \& Moura, DJ 2007, 'Influência das condições ambientais na produção de leite da vacaria Mitra', Revista de Ciências Agrárias, vol. 30, num. 1, p. 143-149.

Ricci, G, Orsi, AM \& Domingues, PF 2013, 'Estresse calórico e suas interferências no ciclo de produção de vacas de leite - Revisão', Revista Veterinária e Zootecnia, vol. 20, num. 3, p. 381-390.

Rodrigues, AL, Souza, B \& Pereira Filho, JM 2010, 'Influência do sombreamento e dos sistemas de resfriamento no conforto térmico de vacas leiteiras', Agropecuária Cientifica no Semi-Árido, vol. 6, num. 2, p. 14-22.

Rosenberg, NJ, Blad, BL \& Verma, SB 1983, 'Microclimate: the biological environment'. 2. ed. New York: Wiley Interscience Publication, 495p.

Santos, DS, Arce, AIC, Piza, LV, Sousa Silva, AC, Costa, EJX \& Tech, ARB 2016 'Redes bluetooth associadas a redes neurais artificiais para monitoramento de suínos', Archivos de Zootecnia, vol. 65, num. 252, p. 557-563.

Souza, BB \& Batista, NL 2012, 'Os efeitos do estresse térmico sobre a fisiologia animal', Agropecuária Científica no Semi-Árido, vol. 8, num. 3, p. 06-10.

Souza, BB, Silva, IJO, Mellace, EM, Santos, RFS, Zotti, CA \& Garcia, PR 2010, 'Avaliação do ambiente físico promovido pelo sombreamento sobre o processo termorregulatório em novilhas leiteiras', Agropecuária Científica no Semiárido, vol. 6, num. 2, p. 59 - 65.

Souza, BB, Silva, GA \& Silva, EMN 2016, 'Índice de conforto térmico para vacas leiteiras em diferentes microrregiões do estado da Paraíba, Brasil', Journal of Animal Behaviour and Biometeorology, vol. 4, num. 1, p. 12-16.
Terra, LAA \& Passador, JL 2012 'Redes neurais artificiais na previsão da inflação: aplicação como ferramenta de apoio à análise de decisões financeiras em organizações de pequeno porte', Revista de Administração Mackenzie, vol. 13, num. 1, p. 68-86.

Torman, VBL, Coster, R \& Riboldi, J 2012, 'Normalidade de variáveis: métodos de verificação e comparação de testes não-paramétricos por simulação. Revista HCPA, vol. 32, num. 2, p. 227-234.

Turco, SHN, Silva, TGF, Santos, LFC, Ribeiro, PHB, Araúio, GGL, Holanda Jr, EV \& Aguiar, MA 2006, 'Zoneamento bioclimático para vacas leiteiras no Estado da Bahia', Revista Engenharia Agrícola, vol. 26, num. 1, p. 20-27.

Valente, GFS, Guimarães, DC, Gaspardi, ALA \& Oliveira, LA 2014, 'Aplicação de redes neurais artificiais como teste de detecção de fraude de leite por adição de soro de queijo', Revista do Instituto de Laticínios Cândido Tostes, vol. 69, num. 6, p. 425-432.

Ventura, RV, Silva, MA, Medeiros, TH, Dionello, NL, Madalena, FE, Fridrich, AB, Valente, BD, Santos, GG, Freitas, LS, Wenceslau, RR, Felipe, VPS \& Corrêa, GS 2012, 'Uso de redes neurais artificiais na predição de valores genéticos para peso aos 205 dias em bovinos da raça Tabapuã', Arquivo Brasileiro de Medicina Veterinária e Zootecnia, vol. 64, num. 2, p. $411-418$.

Wu, CL, Chau, KW \& Fan, C 2010, 'Prediction of Rainfall Time Series Using Modular Artificial Neural Networks Coupled with Data-Preprocessing Techniques', Journal of Hydrology, v.389, nm. 1, p. 146-167.

Yasar, A, Simsek, E, Bilgili, M \& Yucel, A 2012, 'Estimation of relative humidity based on artificial neural network approach in the Aegean Region of Turkey', Meteorology and Atmospheric Physics, vol. 115, num. 1-2, p. 81-87. 